Kallmeyer

Umwandlungsgesetz 



\section{Kallmeyer \\ Umwandlungsgesetz}

Kommentar

\section{Verschmelzung, Spaltung und Formwechsel bei Handelsgesellschaften}

bearbeitet von

Dr. Sebastian Blasche Notarassessor, Köln

Dr. Dirk Dirksen † Rechtsanwalt in Hamburg

Dr. Harald Kallmeyer Rechtsanwalt in Berlin

Dr. Ingo Klöcker, M.C.J. (Austin/Texas) Rechtsanwalt und Notar in Frankfurt/M.

Dr. Dirk Kocher, LL.M. (Helsinki) Rechtsanwalt in Hamburg

Reinhard Marsch-Barner Prof. Dr. Rechtsanwalt in Frankfurt/M. Honorarprofessor an der Universität Göttingen
Dr. Burkhardt W. Meister, LL.M. (Harvard) Notar a.D., Rechtsanwalt in Frankfurt/M.

Dr. Welf Müller

Rechtsanwalt, Wirtschaftsprüfer und Steuerberater in Frankfurt/M

Dr. Mirko Sickinger, LL.M. (Columbia University)

Rechtsanwalt in Köln

Prof. Dr.

Heinz Josef Willemsen

Rechtsanwalt in Düsseldorf Honorarprofessor an der Ruhr-Universität Bochum

Dr. Norbert Zimmermann, LL.M. (Harvard) Notar in Düsseldorf

5. neu bearbeitete und erweiterte Auflage

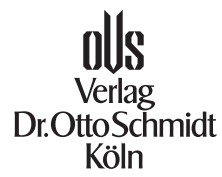




\section{Zitierempfehlung:}

Bearbeiter in Kallmeyer, UmwG, 5. Aufl. 2013, § ... Rz....

Bibliografische Information

der Deutschen Nationalbibliothek

Die Deutsche Nationalbibliothek verzeichnet diese

Publikation in der Deutschen Nationalbibliografie; detaillierte bibliografische Daten sind im Internet über http://dnb.d-nb.de abrufbar.

Verlag Dr. Otto Schmidt KG

Gustav-Heinemann-Ufer 58, 50968 Köln

Tel. 0221/93738-01, Fax 02 21/93738-943

info@otto-schmidt.de

www.otto-schmidt.de

ISBN 978-3-504-37023-7

C2013 by Verlag Dr. Otto Schmidt KG, Köln

Das Werk einschließlich aller seiner Teile ist urheberrechtlich geschützt. Jede Verwertung, die nicht ausdrücklich vom Urheberrechtsgesetz zugelassen ist, bedarf der vorherigen Zustimmung des Verlages. Das gilt insbesondere für Vervielfältigungen, Bearbeitungen, Übersetzungen, Mikroverfilmungen und die Einspeicherung und Verarbeitung in elektronischen Systemen.

Das verwendete Papier ist aus chlorfrei gebleichten Rohstoffen hergestellt, holz- und säurefrei, alterungsbeständig und umweltfreundlich.

Einbandgestaltung: Jan P. Lichtenford, Mettmann

Satz: Schäper, Bonn

Druck und Verarbeitung: Kösel, Krugzell

Printed in Germany 